Cahiers d'études africaines

206-207|2012

L'islam au-delà des catégories

\title{
Buob, Baptiste. - La dinanderie de Fès
}

Constant Hamès

\section{OpenEdition}

Journals

Édition électronique

URL : http://journals.openedition.org/etudesafricaines/14384

DOI : 10.4000/etudesafricaines.14384

ISSN : $1777-5353$

Éditeur

Éditions de l'EHESS

Édition imprimée

Date de publication : 1 juin 2012

Pagination : 693-695

ISBN : 978-2-7132-2349-5

ISSN : 0008-0055

\section{Référence électronique}

Constant Hamès, "Buob, Baptiste. - La dinanderie de Fès », Cahiers d'études africaines [En ligne], 206-207 | 2012, mis en ligne le 04 juin 2012, consulté le 24 septembre 2020. URL : http://

journals.openedition.org/etudesafricaines/14384; DOI : https://doi.org/10.4000/etudesafricaines. 14384

Ce document a été généré automatiquement le 24 septembre 2020.

(c) Cahiers d'Études africaines 


\title{
Buob, Baptiste. - La dinanderie de Fès
}

\author{
Constant Hamès
}

\section{RÉFÉRENCE}

Buов, Baptiste. - La dinanderie de Fès. Un artisanat traditionnel dans les temps modernes. Une anthropologie des techniques par le film et le texte. Paris, Ibis Press-Éditions de la Maison des sciences de l'homme, 2009, 419 p., bibl., ill., DVD.

1 On trouvera surtout dans cet énorme ouvrage une analyse extrêmement détaillée de la fabrication d'objets en cuivre et en laiton plus ou moins argentés dont le paradigme et l'essentiel de la production est représenté par le service à thé. Dans une démarche que naguère on aurait qualifié de marxiste, l'auteur met en relation la fabrication avec les rapports sociaux qui lui sont liés. On obtient alors une véritable somme sur la pratique sub-actuelle et actuelle d'un métier ou d'un ensemble de sous-métiers qui devrait fournir un point de départ pour des recherches locales nouvelles et, soit dans le temps soit dans l'espace du monde musulman, pour des comparaisons inédites.

2 L'examen de l'histoire de l'artisanat de la dinanderie («travail du cuivre et de ses dérivés ", note 1, p. 21) ne permet d'ailleurs pas de comparaisons dans le temps, même si le cuivre a été travaillé de longue date au Maroc pour le mobilier religieux ou palatial. Cela est dû au fait que la dinanderie marocaine ne s'est constituée qu'avec l'importation et la diffusion du thé qui commencent vers les années 1870, pour se généraliser surtout au début $\mathrm{du} \mathrm{XX}^{\mathrm{e}}$ siècle, pratiquement au début du protectorat français. L'auteur, qui utilise de façon remarquable le vocabulaire arabe local dans toutes ses analyses, aurait d'ailleurs pu indiquer ici que le terme dialectal etèy ou atay n'est qu'une prononciation locale du terme franco-anglais thé, tandis que l'arabe classique, lui, reprend le terme chinois d'origine, shay.

La «traditionnalisation» de la fabrication de ces objets artisanaux, somme toute récents et au surplus copiés sur les modèles anglais (théière notamment), à partir de métaux eux aussi importés (laiton, maillechort - variante argentée de laiton - cuivre), forme la toile de fond de la démonstration de l'ouvrage et justifie son premier sous- 
titre: «un artisanat traditionnel dans les temps modernes", à condition de ne pas oublier le caractère récent de sa mise en place. À partir de ce constat, on s'enfonce dans l'analyse minutieuse de la corporation des dinandiers de la ville ancienne de Fès $\left(2^{\mathrm{e}}\right.$ partie : «Objets, espace et acteurs»), en découvrant leurs clientèles et surtout leur organisation interne, à la fois spatiale (ateliers, boutiques) et sociale (hiérarchie de métiers et de fonctions). On retiendra, entre autres, que la division du travail ainsi que l'accès inégal au capital financier ont créé des inégalités considérables entre les riches fournisseurs de matière première et, au bout de l'échelle de production, l'ouvrier à peine spécialisé dont le statut professionnel n'évoluera guère, même si ses connaissances et son savoir-faire augmentent. «Enfin, note l'auteur, il faudrait ajouter à ces chiffres [de travailleurs de la profession], le nombre impressionnant d'enfants non pris en compte dans le recensement [...]; ce changement se traduit au plan du vocabulaire puisqu'on parle aujourd'hui plus souvent d'enfants (drâri) que d'apprentis (mt'allemîn) »(p. 162).

4 En suivant, comme le fait fort bien B. Buob, le vocabulaire désignant les artisans, on découvre leur dénomination ancienne de seffâra ou saffârîn (de la racine arabe « couleur jaune ») qui a donné, à Fès, son nom au territoire corporatif des artisans du cuivrelaiton, rue ou place Seffarine. Leur appellation plus récente et actuelle, de swâiniya (pluriel) dont l'origine provient de Sin = Chine, semble faire référence à des objets en porcelaine s'étant transformés par la suite en cuivre-laiton. Une autre appellation retient l'attention, celle de m'allem, de la racine arabe enseigner, qui désigne l'artisan expérimenté transmettant son savoir à des apprentis ( $\left.m t^{\prime} a l l e m\right)$ et entouré d'autres ouvriers (sâna', de la racine arabe fabriquer). Cette dernière catégorie socio-linguistique se réfère à une organisation sociale du travail qui n'a plus vraiment cours au moment de l'enquête car le processus de production du nécessaire à thé s'est taylorisé et financiarisé, faisant éclater la cellule unique de l'atelier avec maître et apprentis. Mais il est intéressant de noter, au-delà du cas de Fès et du Maroc, que le terme m'allem (pluriel m'allemin), s'est appliqué, au Sahara de l'Ouest, à tous les artisans polyvalents et à leurs femmes (m'allema), de concert avec le terme sâna', tous deux frappés d'un sens socialement péjoratif, propre aux sociétés saharo-ouest-africaines mais dont la permanence et l'étendue concernent, à des degrés divers, tout le monde arabomusulman qui déconsidère certaines professions manuelles sous des prétextes socioreligieux variés.

5 L'auteur aurait pu investiguer dans ce sens en s'aidant notamment du petit article novateur de R. Brunschvig "Métiers vils en islam $~^{1}$. On trouvera dans cette partie de l'ouvrage également des éléments historiques qui permettront peut-être de comprendre, du moins en partie, pourquoi les artisans du désert du Sahara de l'Ouest sont légendairement crédités d'une origine juive (voir p. 42 et s.).

On ne tentera pas de rendre compte de l'infinie précision du relevé des techniques de travail, au sens large et étroit à la fois, consignées avec beaucoup d'application et de pédagogie, notamment à travers les illustrations. Un des éléments notables qui rend compte de la situation actuelle des artisans du thé, tient dans l'évolution technologique et financière des procédés de fabrication. Les capitaux et les machines ont changé l'artisanat et fait voler en éclats le caractère holistique du travail artisanal du ma'allem de jadis. Mais celui-ci s'est maintenu dans certaines phases (ciselure par exemple) de la chaîne de fabrication. 
7 L'aspect documentaire précis de l'analyse des techniques n'aurait certainement pas été possible sans l'utilisation judicieuse de l'enregistrement filmé, ainsi que l'indique le deuxième sous-titre : «Une anthropologie des techniques par le film et le texte. » Les références à Leroi-Gourhan sont ici importantes. Le livre est d'ailleurs fort utilement accompagné d'un DVD qui restitue à travers quatre cas d'espèce les découpages analytiques de l'investigation. Une annexe explique aussi les partis pris méthodologiques impliqués par les films.

8 L'ouvrage est une somme sur la situation sociale, sur les métiers et les techniques de quelques milliers de professionnels et d'auxiliaires attachés à la fabrication des ustensiles du "nécessaire à thé » dans la ville de Fès. On regrettera simplement qu'il manque à cet outil de travail un index qui aurait incorporé notamment la très riche documentation linguistique arabe relevée sur le terrain.

9 1. R. BRUNSCHVIG, "Métiers vils en islam ", in R. BRUnSChVIG (dir.), Études d’islamologie, t. 1, G. P. Maisonneuve et Larose, 1976, pp. 145-164. 